\title{
Salivary Gland Infection, CTCAE
}

National Cancer Institute

\section{Source}

National Cancer Institute. Salivary Gland Infection, CT CAE. NCI Thesaurus. Code C143822.

A disorder characterized by an infectious process involving the salivary gland. 